01;04

\title{
Поперечное движение частицы с осциллирующим зарядом и переменной массой в магнитном поле
}

\author{
(C) 3.3. Алисултанов ${ }^{1,2}$, Г.Б. Рагимханов ${ }^{2}$
}

${ }^{1}$ Институт фризики им. Х.И. Амирханова ДагНЦ РАН, Махачкала, Россия

2 Дагестанский государственный университет, Махачкала, Россия

E-mail: zaur0102@gmail.com

Поступило в Редакцию 27 апреля 2017 г.

В окончательной редакции 21 ноября 2017г.

Решена задача движения частицы с осциллирующим электрическим зарядом и изменяющейся массой в однородном магнитном поле. Рассмотрены три закона изменения массы: линейный рост, осцилляции и ступенчатый рост. Получены аналитические выражения для скорости частицы при различных временны́х зависимостях массы частицы. Установлено, что одновременный учет изменения массы и заряда приводит к существенному изменению траектории частиц.

DOI: 10.21883/PJTF.2018.05.45704.16839

Пылевая плазма, т.е. плазма, в которой взвешены электрически заряженные микрочастицы конденсированного вещества, является объектом интенсивных экспериментальных и теоретических исследований (см., например, [1-3]). Разнообразие форм существования и свойств пылевой плазмы позволило обнаружить ряд новых эффектов и явлений, некоторые из которых описаны в работах [4-7].

Известно [8], что в переменном электрическом поле под действием так называемой силы Гапонова-Миллера заряженная частица, совершая осцилляции, может дрейфовать в некотором определенном направлении, т. е. положение, относительно которого частица совершает колебания, со временем может смещаться. Следует отметить, что пылинка, движущаяся в плазме, приобретает электрический заряд за счет поглощения ее поверхностью электронов и ионов. При этом нестационарные плазменные процессы приводят к тому, что заряд пылинки изменяется во времени. 
Законы движения точечной частицы, заряд которой изменяется во времени, изучались во многих работах, например в [9-18]. Известно, что в процессе распространения в плазме волны $[15,16,18]$ заряд пылинки осциллирует во времени с частотой волны $\Omega$. Однако в плазменных средах возможен и более сложный характер изменения заряда частицы, например скачком [17], соответственно и характер движения будет разнообразным. В частности, в $[19,20]$ удалось установить, что на гранулу со стороны интенсивной быстро осциллирующей ионно-звуковой волны действует пондеромоторная сила, составляющие которой пропорциональны волновому вектору и кубу амплитуды поля. Эти составляющие приводят к направленному транспорту пылевой фракции плазмы.

В то же время в работах [11-14] на основе вычислительного эксперимента исследованы различные кинетические характеристики пылинок микронных размеров, помещенных в плазму. Показано, что скорость изменения заряда частицы зависит от ее начальной массы, причем чем больше начальная масса, тем быстрее идет процесс зарядки и тем больше максимальное значение заряда в момент установления стационарного значения.

Таким образом, кинетика процессов с участием микрочастиц в плазме определяется также массой частицы и ее размерами. В то же время, вообще говоря, масса частицы в пылевой плазме не будет постоянной величиной за счет прилипания или отщепления других микрочастиц. Особенно такого рода нестационарные эффекты важны в системах типа фуллереновой плазмы. В связи с этим представляет интерес исследовать законы движения частицы с переменной массой во внешних полях.

Рассмотрим задачу в следующей геометрической постановке. Пусть частица вылетает из начала координат с начальной скоростью $v_{x 0}$, перпендикулярной магнитному полю $\mathbf{H}=(0,0, H)$. Уравнения движения с осциллирующим зарядом и переменной массой запишутся в виде

$$
\begin{gathered}
\frac{d v_{x}}{d t}+\frac{1}{m(t)} \frac{d m(t)}{d t} v_{x}=\frac{H}{m(t) c}\left(q_{0}+q_{1} \cos (\Omega t)\right) v_{y}, \\
\frac{d v_{y}}{d t}+\frac{1}{m(t)} \frac{d m(t)}{d t} v_{y}=-\frac{H}{m(t) c}\left(q_{0}+q_{1} \cos (\Omega t)\right) v_{x} .
\end{gathered}
$$

Письма в ЖТФ, 2018, том 44, вып. 5 
Решение будем искать в виде $v=v_{x}+i v_{y}$. С помощью этих уравнений легко показать, что

$$
\frac{d v}{d t}=-\left[\frac{1}{m(t)} \frac{d m(t)}{d t}+i \frac{H}{m(t) c}\left(q_{0}+q_{1} \cos (\Omega t)\right)\right] v .
$$

Отсюда

$$
v=v_{0} \exp \left(-\int \frac{1}{m(t)} \frac{d m(t)}{d t} d t\right) \exp \left\{-i \int \frac{H}{m(t) c}\left(q_{0}+q_{1} \cos (\Omega t)\right) d t\right\} .
$$

Далее заметим, что

$$
\int \frac{1}{m(t)} \frac{d m(t)}{d t} d t=\int \frac{d m(t)}{m(t)}=\ln \frac{m(t)}{m_{0}} .
$$

Таким образом,

$$
\begin{aligned}
& v_{x}=\frac{v_{0} m_{0}}{m(t)} \cos \left\{\int \frac{H}{m(t) c}\left(q_{0}+q_{1} \cos (\Omega t)\right) d t\right\}, \\
& v_{y}=-\frac{v_{0} m_{0}}{m(t)} \sin \left\{\int \frac{H}{m(t) c}\left(q_{0}+q_{1} \cos (\Omega t)\right) d t\right\} .
\end{aligned}
$$

Мы будем рассматривать три типа временно́й эволюции массы.

1. Линейный рост: $m(t)=m_{0}+A t$, где $A=d m / d t-$ скорость роста массы.

2. Осцилляции: $m(t)=m_{0}+B \sin \Omega t, B-$ амплитуда осцилляций массы.

3. Ступенчатый рост: $m(t)=m_{0}+C \Sigma_{n=1}^{\infty} \Theta\left(t-n t_{0}\right)$, где $C-$ высота ступеньки (величина скачка массы), $t_{0}$ - ширина ступеньки (период скачка массы).

Соответственно для трех случаев имеем: для первого случая

$$
\begin{aligned}
& v_{x}=\frac{v_{0}}{1+\frac{A}{m_{0}} t} \cos \left[I_{1}(t)\right], \\
& v_{y}=-\frac{v_{0}}{1+\frac{A}{m_{0}} t} \sin \left[I_{1}(t)\right],
\end{aligned}
$$

Письма в ЖТФ, 2018, том 44, вып. 5 


$$
\begin{aligned}
I_{1}= & \frac{H q_{0}}{A c}\left\{\ln \left|1+\frac{A}{m_{0}} t\right|+\frac{q_{1}}{q_{0}}\left[\cos \frac{\Omega m_{0}}{A} \operatorname{ci}\left(\frac{\Omega}{A}\left(m_{0}+A t\right)\right)\right.\right. \\
& \left.\left.+\sin \frac{\Omega m_{0}}{A} \operatorname{si}\left(\frac{\Omega}{A}\left(m_{0}+A t\right)\right)\right]\right\},
\end{aligned}
$$

где $\operatorname{ci}(x)=-\int_{x}^{\infty} \frac{\cos t}{t} d t$ и $\operatorname{si}(x)=\int_{0}^{x} \frac{\sin t}{t} d t-$ интегральные косинус и синус соответственно; для второго случая

$$
\begin{gathered}
v_{x}=\frac{v_{0}}{1+\frac{B}{m_{0}} \Omega t} \cos \left[I_{2}(t)\right], \\
v_{y}=-\frac{v_{0}}{1+\frac{B}{m_{0}} \sin \Omega t} \sin \left[I_{2}(t)\right],
\end{gathered}
$$

$I_{2}(t)=\frac{H}{c \Omega} \times$

$$
\times\left[\begin{array}{c}
\frac{q_{0}}{m_{0}} \frac{2}{\sqrt{1-\frac{B^{2}}{m_{0}^{2}}}} \arctan \frac{\tan \frac{\Omega t}{2}+\frac{B}{m_{0}}}{\sqrt{1-\frac{B^{2}}{m_{0}^{2}}}}+\frac{q_{1}}{B} \ln \left|1+\frac{B}{m_{0}} \sin \Omega t\right| \\
\frac{q_{0}}{m_{0}} \frac{1}{\sqrt{\frac{B^{2}}{m_{0}^{2}}-1}} \ln \frac{\tan \frac{\Omega t}{2}+\frac{B}{m_{0}}-\sqrt{\frac{B^{2}}{m_{0}^{2}}-1}}{\tan \frac{\Omega t}{2}+\frac{B}{m_{0}}+\sqrt{\frac{B^{2}}{m_{0}^{2}}-1}}+\frac{q_{1}}{B} \ln \left|1+\frac{B}{m_{0}} \sin \Omega t\right|
\end{array}\right] \quad m_{0}^{2}>B^{2},
$$

для третьего случая

$$
\begin{gathered}
u_{x}=\frac{v_{0}}{1+\frac{C}{m_{0}} \sum_{n=1}^{\infty} \Theta\left(t-n t_{0}\right)} \cos \left[I_{3}(t)\right], \\
v_{x}=-\frac{v_{0}}{1+\frac{C}{m_{0}} \sum_{n=1}^{\infty} \Theta\left(t-n t_{0}\right)} \sin \left[I_{3}(t)\right], \\
I_{3}(t)=\frac{H}{c} \frac{1}{m_{0}+C \sum_{n=1}^{\infty} \Theta\left(t-n t_{0}\right)}\left(q_{0} t+\frac{q_{1}}{\Omega} \sin (\Omega t)\right) .
\end{gathered}
$$




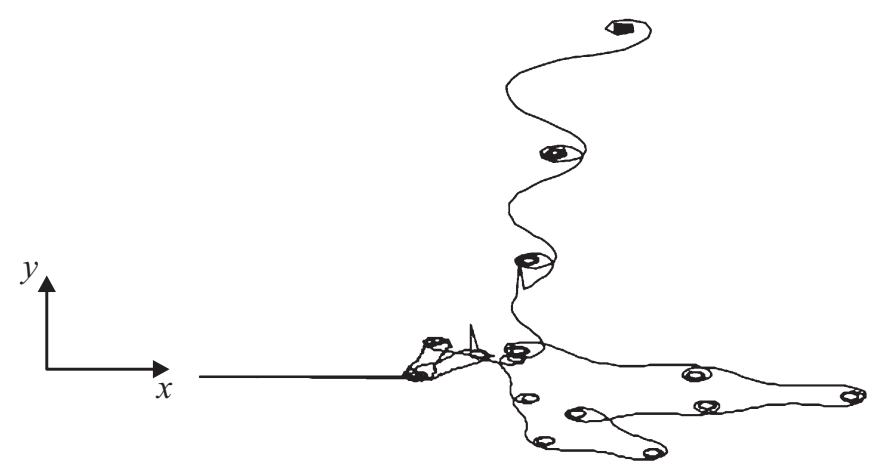

Рис. 1. Траектория движения частицы с линейным ростом массы с $A=10^{-8} \mathrm{~g} / \mathrm{s}$, $m_{0}=10^{-7} \mathrm{~g} / \mathrm{s}, \omega_{1} / \omega_{0}=15 / 12$.

Построение траектории частицы может быть выполнено с помощью интегрирования

$$
\left\{\begin{array}{l}
x=\int_{0}^{t} v_{x}(t) d t \\
y=\int_{0}^{t} v_{y}(t) d t
\end{array}\right.
$$

На рис. 1-3 показаны траектории движения частицы для указанных выше трех случаев временно́й зависимости массы. Траектории для случая постоянной массы приведены в работе [18].

Рассмотрим результаты анализа траекторий частицы для различных вариантов изменения массы. Далее везде, как и в работе [18], используются следующие обозначения: $\omega_{0}=g_{0} H / m c, \omega_{1}=q_{1} H / m c$. Как видно из рисунков, в случае переменной массы траектории существенно отличаются от полученных в случае постоянной массы, причем форма траектории достаточно сильно зависит от закона временно́й эволюции. Опуская детальный анализ этих траекторий, который аналогичен проведенному в работе [18], отметим лишь следующее. В зависимости от закона изменения массы может реализоваться случай финитного или инфинитного движения. Кроме того, даже в рамках одного закона изменения массы для разных значений параметров наблюдается различный характер движения: для некоторых параметров частица

Письма в ЖТФ, 2018, том 44, вып. 5 

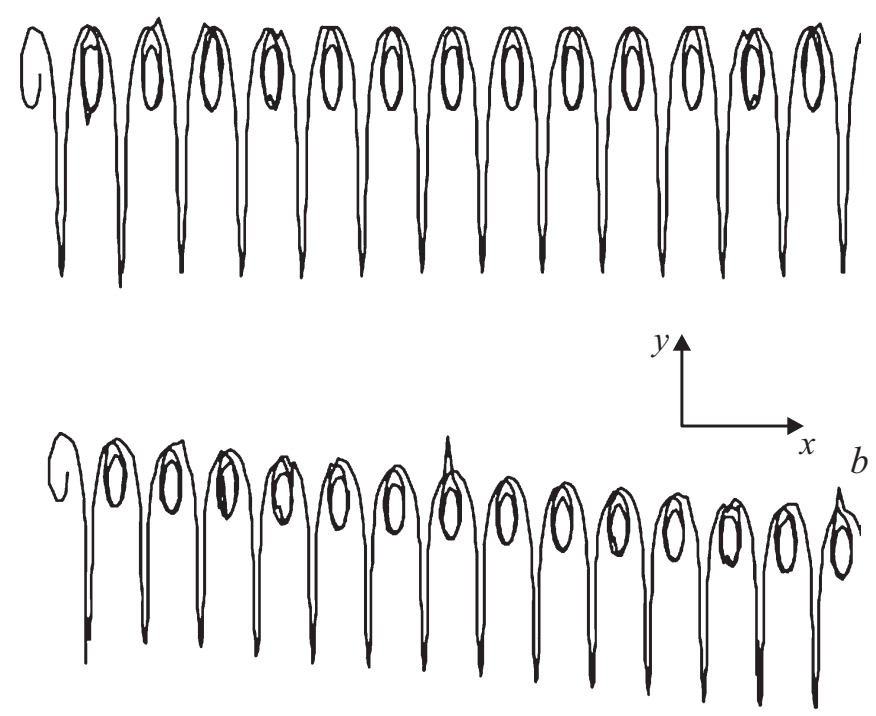

Рис. 2. Траектории движения частицы с осциллирующей массой для значений параметров $B=10^{-11} \mathrm{~g}, m_{0}=10^{-7} \mathrm{~g}, \Omega / \omega_{0}=5 / 12, \omega_{1} / \omega_{0}=15 / 12 . a$ - масса частицы постоянна, $b-$ масса частицы осциллирует.

испытывает неограниченное поперечное дрейфовое движение, а для других параметров частица движется по финитной траектории, целиком лежащей в некоторой кольцевой области.

В случае линейного роста массы частицы испытывают неограниченное поперечное дрейфовое движение даже при очень слабой скорости роста массы частицы. В случае осцилляции массы частица совершает поперечный дрейф.

Таким образом, в работе решена задача движения частицы с осциллирующим электрическим зарядом и переменной массой в магнитном поле. Установлено, что частица с осциллирующим электрическим зарядом и переменной массой может испытывать поперечный дрейф в однородном магнитном поле. Рассмотрены различные случаи изменения массы частицы. Учет изменения массы приводит к существенному изменению формы траектории. Обнаружено, что в случае изменения 


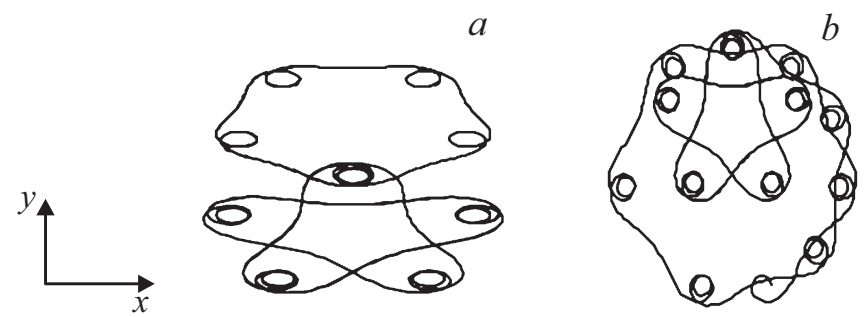

Рис. 3. Траектории движения частицы со ступенчатым ростом массы для значений параметров $m_{0}=10^{-7} \mathrm{~g}, t_{0}=10^{-6} \mathrm{~s}, C=10^{-9}$ (a) и $10^{-10} \mathrm{~g}(b)$. $\omega_{1} / \omega_{0}=15 / 12$.

массы ступенчатым образом все же возможно финитное движение частицы. В заключение отметим, что в реальной системе на разных временны́х промежутках могут реализоваться разные типы зависимости массы от времени, а общая картина будет достаточно сложной.

Работа выполнена при частичной поддержке грантов Президента РФ МК-2130.2017.2, РФФИ № 15-02-03311а, главы РД (2016).

\section{Список литературы}

[1] Tomas H., Morfill G.E. // Nature (London). 1996. V. 379. P. 806-809.

[2] Цытович В.Н. // УФН. 2015. Т. 185. № 2. С. 161-179.

[3] Игнатов А.М. // Физика плазмы. 1998. Т. 24. № 8. С. 731-737.

[4] Цитович В.Н. // УФН. 1997. Т. 167. № 1. С. 57-99.

[5] Verheest F. // Space Sci. Rev. 1997. V. 77. P. 267-302.

[6] Verheest F. // Plasm. Phys. Control. Fusion. 1999. V. 41. N 3A. P. A445-A452.

[7] Merlino R.L., Goree J.A. // Phys. Todey. 2004. V. 57. N 7. P. 32-38.

[8] Shukla P.K., Rosenberg M. // Phys. Plasmas. 1999. V. 6. N 4. P. 1371-1373.

[9] Dikarev V.V. // Astron. Astrophys. 1999. V. 346. N 3. P. 1011-1019.

[10] Howard J.E. // Phys. Lett. A. 2007. V. 366. N 1-2. P. 91-96.

[11] Владимиров С.В., Крамер Н.Ф., Майоров С.А. // Краткие сообщения по физике ФИАН. 2000. В. 10. С. 7-13.

[12] Maiorov S.A., Vladimirov S.V., Cramer N.F. // Phys. Rev. E. 2001. V. 63. N 1. P. 017401 (1-4).

[13] Vladimirov S.V., Maiorov S.A., Cramer N.F. // Phys. Rev. E. 2001. V. 63. N 6. P. $067401(1-4)$.

Письма в ЖТФ, 2018, том 44, вып. 5 
[14] Майоров С.A. // Физика плазмы. 2002. Т. 28. № 10. С. 946-952.

[15] Старостин А.Н., Петрушевич Ю.В. // Физика плазмы. 2005. Т. 31. № 3. C. 233-239.

[16] Дубинов А.Е., Сазонкин М.А. // ЖТФ. 2008. Т. 78. В. 9. С. 29-40.

[17] Meyer-Vernet N. // Astron. Astrophys. 1982. V. 105. N 1. P. 98-106.

[18] Дубинов А.Е. // Письма в ЖТФ. 2011. Т. 37. В. 3. С. 59-63.

[19] Rosenberg M. // Phys. Scripta. 2010. V. 82. N 3. P. 035505 (1-7).

[20] Ломтев А.И. // ЖТФ. 2014. Т. 84. В. 3. С. 130-132. 